\title{
Proteomic Investigation of Dermal Fibroblasts Isolated from Affected and Unaffected Skin Samples from Patients with Limited Cutaneous Systemic Sclerosis: 2 Distinct Entities?
}

\author{
Claudio Corallo, Annalisa Santucci, Giulia Bernardini, Natale Figura, Roberto Leoncini, \\ Giulia Riolo, Antonio Montella, Chiara Chirico, Ranuccio Nuti, and Nicola Giordano
}

\begin{abstract}
Objective. To identify using proteomic analysis the proteins of altered abundance in the affected and unaffected limited cutaneous systemic sclerosis ( $1 \mathrm{cSSc})$ skin fibroblasts.

Methods. Excision biopsies ( $3 \mathrm{~mm}$ ) were obtained from the affected and unaffected skin of 5 patients with lcSSc. Dermal fibroblasts were isolated enzymatically. Two-dimensional gel electrophoresis was used to separate and define proteins in affected and unaffected fibroblast lysates. Proteins of altered abundance were identified by mass spectrometry. Differences among skin samples were confirmed also by immunohistochemistry (IHC) and by quantitative real-time PCR (qRT-PCR) for type I collagen (Col-1) and vimentin (VIM).
\end{abstract}

Results. Proteomic analysis revealed different expressions of proteins involved in cytoskeleton organization $(27 \%)$, extracellular matrix remodeling $(11 \%)$, response to oxidative stress $(22 \%)$, energy metabolism (19\%), protein metabolism $(5 \%)$, cellular homeostasis $(5 \%)$, signal transduction $(3 \%)$, and protein transcription, synthesis, and turnover (8\%). IHC analysis showed that SSc-affected epidermis is thickened and the dermis is strongly reactive to Col-1 and VIM (typical markers of activated myofibroblasts) compared to SSc-unaffected skin, whose stainings are comparable to those of control healthy skin. Overexpression of Col-1 and VIM mRNA levels in affected lcSSc fibroblasts compared to unaffected lcSSc ones was confirmed by qRT-PCR.

Conclusion. Consistent with previous studies, these findings are important for 2 reasons: first, because they reveal the opposite behavior of dermal fibroblasts in the unaffected and affected skin areas of the same patient with lcSSc; second, because they demonstrate the histological/histochemical similarities between unaffected skin from patients with $1 \mathrm{cSSc}$ and healthy control skin. (First Release December 1 2016; J Rheumatol 2017;44:40-8; doi:10.3899/jrheum.160736)

Key Indexing Terms:

SYSTEMIC SCLEROSIS PROTEOMICS FIBROSIS DERMAL FIBROBLASTS

2-D ELECTROPHORESIS PROTEOMICS OF SCLERODERMIC FIBROBLASTS

Systemic sclerosis ( $\mathrm{SSc}$ ) is an autoimmune connective tissue disease characterized by vascular injury and fibrosis of the

From the Department of Medicine, Surgery, and Neurosciences; the Department of Biotechnology, Chemistry and Pharmacy; and the Department of Medical Biotechnology, University of Siena, Siena, Italy.

C. Corallo, PhD, Department of Medicine, Surgery and Neurosciences, University of Siena; A. Santucci, PhD, Department of Biotechnology, Chemistry and Pharmacy, University of Siena; G. Bernardini, PhD, Department of Biotechnology, Chemistry and Pharmacy, University of Siena; N. Figura, MD, Department of Medicine, Surgery, and Neurosciences, University of Siena; R. Leoncini, PhD, Department of Medical Biotechnology, University of Siena; G. Riolo, PhD, Department of Medical Biotechnology, University of Siena; A. Montella, MD, Department of Medicine, Surgery, and Neurosciences, University of Siena; C. Chirico, MD, Department of Medicine, Surgery, and Neurosciences, University of Siena; R. Nuti, MD, Department of Medicine, Surgery, and Neurosciences, University of Siena; N. Giordano, MD, Department of Medicine, Surgery, and Neurosciences, University of Siena.

Address correspondence to C. Corallo, Scleroderma Unit, Department of Medicine, Surgery, and Neurosciences, University of Siena, 53100 Siena, Italy.E-mail: corallo.claudio@gmail.com

Accepted for publication October 18, 2016. skin and internal organs ${ }^{1}$. Subsets of SSc are limited cutaneous SSc (lcSSc), diffuse cutaneous SSc (dcSSc), and SSc without skin involvement ${ }^{2}$. Persistent fibroblast activation is central to pathogenesis, and SSc fibroblasts have increased ability to secrete, adhere to, and contract extracellular matrix ${ }^{3}$, although the initiating factors leading to fibroblast activation are not completely understood. Changes in the dermis have received much attention in SSc, because overproduction of extracellular matrix by dermal fibroblasts accounts for much of the pathological skin thickening and scarring seen in the disease ${ }^{4}$. The clinical semiquantitative assessment of skin thickness by palpation, modified Rodnan skin score (mRSS), is considered the gold standard and often the only primary outcome measure used in clinical trials of SSc disease-modifying agents ${ }^{1,2}$. This subjective and highly variable assessment is fraught with inaccuracies, as pointed out in the literature ${ }^{5}$. Therefore, it is generally accepted that the development of objective and reliable markers reflecting

Personal non-commercial use only. The Journal of Rheumatology Copyright $\odot$ 2016. All rights reserved. 
the severity of skin fibrosis would be of great value for improving the performance of clinical trials and the accurate assessment of the efficacy of a given treatment ${ }^{6}$. In our present study, we describe the results of a proteomic analysis of the lysates of fibroblasts isolated from the affected and unaffected skin areas of the same patient with lcSSc. The proteomic analysis allowed the identification of several proteins differently expressed by the 2 fibroblast populations, reflecting the increased fibrogenesis of those belonging to affected skin areas. In fact, thanks to the first proteomic screening, we selected a couple of unaffected and affected lcSSc skin samples for the presence of both protein (immunohistochemistry) and mRNA levels [quantitative real-time PCR (qRT-PCR)] of those putative biomarkers: they may be useful to assess the extent and severity of the SSc fibrotic process and contribute to better understanding the pathogenetic mechanism of the disease.

\section{MATERIALS AND METHODS}

Skin biopsy and fibroblast isolation and culture. Patients selected for inclusion in the study were women from the lcSSc subset age $55 \pm 8.6$ (mean $\pm \mathrm{SD}$ ) years, with disease duration $6 \pm 3.9$ years. Ethical approval was obtained for the study from the ethics committee of the University of Siena (n. CELAOUS28022012) in accordance with the Helsinki Declaration of 1975, and patients gave written informed consent to participate in the study. The 3-mm excision biopsies were obtained from the affected skin (third finger, mRSS graded 2 at biopsy site) and from unaffected skin (mid-forearm, mRSS graded 0 at biopsy site). The lcSSc unaffected skin was defined by both clinical palpation and by histological examination that excluded SSc-related lesions. Biopsies were taken prior to commencement of immunosuppressive or disease-modifying treatments. The biopsy samples, processed within $1 \mathrm{~h}$ of excision, were divided in half: 1 for histopathological and immunohistochemical analysis, and the other for establishment of dermal fibroblast cell strains. For this purpose, fibroblasts were isolated from skin specimens by enzymatic digestion. Briefly, explants were de-epidermized using a dispase solution (dispase activity $14 \mathrm{U} / \mathrm{ml}$; Sigma-Aldrich) for $2 \mathrm{~h}$ at $37^{\circ} \mathrm{C}$ and then were dissolved into a collagenase III solution ( $2.4 \mathrm{U} / \mathrm{ml}$; Sigma-Aldrich) for $30 \mathrm{~min}$. Fibroblasts obtained were passaged twice and cultured at density of $1 \times 10^{6}$ cells/flask in Dulbecco modified Eagle's medium (all substances Sigma-Aldrich) supplemented with penicillin $(100 \mathrm{U} / \mathrm{ml})$, streptomycin $(100 \mu \mathrm{g} / \mathrm{ml}), 0.25 \mu \mathrm{g} / \mathrm{ml}$ amphotericin B, $2 \mathrm{mM}$ glutamine, and $10 \%$ fetal bovine serum, and incubated at $37^{\circ} \mathrm{C}$ in an atmosphere of $5 \% \mathrm{CO}_{2} 95 \%$ air, till confluence (1 week) onto $75 \mathrm{~cm}^{2}$ flasks (BD Costar). Viability was estimated by trypan blue (Sigma-Aldrich). Fibroblasts were used at third passage for experiments.

Protein extraction and 2-dimensional electrophoresis (2DE). Proteomic profiling was based on skin biopsy material (affected and unaffected fibroblasts) from 5 patients with lcSSc. Dermal fibroblasts were lysed and dissolved in a conventional 2-dimensional lysis buffer [8 M urea, $4 \%(\mathrm{w} / \mathrm{v})$ 3-[(3-cholamidopropyl)-dimethylammonio]-1-propanesulfonate, $40 \mathrm{mM}$ Tris base, $65 \mathrm{mM}$ dithioerythritol, and a trace of bromophenol blue]. Protein concentration was calculated with Bradford assay ${ }^{7}$. The 2DE was carried out for 5 pooled unaffected or 5 pooled affected fibroblast samples using the Immobiline-polyacrylamide system ${ }^{8}$. Isoelectric focusing (IEF) was carried out on a preformed immobilized non-linear $\mathrm{pH}$ gradient, from $\mathrm{pH} 3$ to 10 , 18-cm strips (GE Healthcare), and performed using the Ettan IPGphor system (GE Healthcare). Sample load was $90 \mu \mathrm{g}$ per strip in analytical runs, and $1 \mathrm{mg}$ per strip in mass spectrometry (MS) preparative gels. Analytical strips were rehydrated for $1 \mathrm{~h}$ at $0 \mathrm{~V}$ and for $8 \mathrm{~h}$ at $30 \mathrm{~V}$, at $16^{\circ} \mathrm{C}$.

Then the proteins were focused under the following electrical conditions at $16^{\circ} \mathrm{C}$ : $200 \mathrm{~V}$ for $1 \mathrm{~h}$, from 300 to $3500 \mathrm{~V}$ in $30 \mathrm{~min}, 3500 \mathrm{~V}$ for $3 \mathrm{~h}$, from
3500 to $8000 \mathrm{~V}$ in $30 \mathrm{~min}, 8000 \mathrm{~V}$ up to a total of $80,000 \mathrm{Vh}$. MS-preparative strips were rehydrated with $350 \mu \mathrm{l}$ of lysis buffer and $2 \% \mathrm{v} / \mathrm{v}$ carrier ampholyte at room temperature for $12 \mathrm{~h}$. Sample load was performed using a cup loading method, with the cup applied at the cathodic and anodic ends of the strip. For MS-preparative runs, IEF was performed using the Multiphor II electrophoresis system (GE Healthcare) according to the following voltage steps at $16^{\circ} \mathrm{C}$ : $200 \mathrm{~V}$ for $6 \mathrm{~h}, 600 \mathrm{~V}$ for $1 \mathrm{~h}, 1200 \mathrm{~V}$ for $1 \mathrm{~h}, 3500 \mathrm{~V}$ for $3 \mathrm{~h}, 5000 \mathrm{~V}$ for $14 \mathrm{~h}$. After the first dimension run, the analytical and preparative immobilized $\mathrm{pH}$ gradient gels were equilibrated in $6 \mathrm{M}$ urea, $2 \% \mathrm{w} / \mathrm{v}$ sodium dodecyl sulfate (SDS), $2 \% \mathrm{w} / \mathrm{v}$ dithioerythritol, $30 \% \mathrm{v} / \mathrm{v}$ glycerol, and $0.05 \mathrm{M}$ Tris- $\mathrm{HCl} \mathrm{pH} 6.8$ for $12 \mathrm{~min}$; and for further $5 \mathrm{~min}$ in $6 \mathrm{M}$ urea, $2 \% \mathrm{w} / \mathrm{v} \mathrm{SDS}, 2.5 \% \mathrm{w} / \mathrm{v}$ iodoacetamide, $30 \% \mathrm{v} / \mathrm{v}$ glycerol, $0.05 \mathrm{M}$ Tris- $\mathrm{HCl} \mathrm{pH} 6.8$, and a trace of bromophenol blue. The second dimension was carried out, at $10^{\circ} \mathrm{C}$, on $9 \%-16 \%$ polyacrylamide linear gradient gels $(18 \times 20 \mathrm{~cm} \times 1.5 \mathrm{~mm})$ at $40 \mathrm{~mA} /$ gel constant current, until the dye front reached the bottom of the $\mathrm{gel}^{9}$. Analytical gels were stained with ammoniacal silver nitrate ${ }^{10,11}$. MS-preparative gels were stained according to a silver stained protocol compatible with MS according to the manufacturer's instructions. Silver-stained gels were then digitalized using the Image Scanner III laser densitometer (GE Healthcare). Computer-aided 2-dimensional image analysis was carried out with the Image Master Platinum 7.0 software (GE Healthcare). Spot detection was achieved after defining and saving a set of detection variables, enabling filtering and smoothing of the original gel scans to clarify spots, and removal of vertical and horizontal streaks and speckles. The analysis process was performed by matching the gels of each group with a reference gel named "master" by the software. By this procedure, the Image Master Platinum algorithm matched the other gels to find qualitative and quantitative differences.

Analysis with MS. Protein identification was carried out by peptide mass fingerprinting on an Ultraflex III matrix-assisted laser desorption/ionization time-of-flight (MALDI-TOF/TOF) mass spectrometer (Bruker Daltonics) ${ }^{12}$. Electrophoretic spots from silver-stained gels were manually excised, de-stained, and dehydrated in acetonitrile. Then the spots were rehydrated in trypsin solution (Sigma-Aldrich) and in-gel protein digestion was performed overnight at $37^{\circ} \mathrm{C}$. For each protein digest, $0.75 \mu 1$ was spotted onto the MALDI target and allowed to dry. Then $0.75 \mu$ l of matrix solution [saturated solution of alpha-cyano-4-hydroxycinnamic acid in 50\% (v/v) acetonitrile and $0.5 \%(\mathrm{v} / \mathrm{v})$ trifluoroacetic acid] was applied to the dried sample, and allowed to dry again. Tryptic peptide masses were automatically acquired by the Flex Analysis software (Bruker Daltonics). Mass fingerprinting searching was carried out in Swiss-Prot/TrEMBL database using MASCOT (Matrix Science Ltd.) software available online. Taxonomy was limited to Homo sapiens, mass tolerance was $<100 \mathrm{ppm}$, and the number of accepted missed cleavage sites was set to 1 . Alkylation of cysteine by carbamidomethylation was assumed as fixed modification, while oxidation of methionine was considered as a possible one. The criteria used to accept protein identification were sequence coverage, number of matched peptides, and probabilistic score. For tryptic peptides that did not produce an unambiguous identification, peptide sequencing by tandem mass spectrometry on the Ultraflex III MALDI-TOF/TOF mass spectrometer (Bruker Daltonics) was performed. MS/MS database searching was carried out using Flex Analysis algorithm (Bruker Daltonics) and Mascot MS/MS ion search software in Swiss-Prot/TrEMBL database. Search criteria applied were MS mass accuracy \pm 1.2 Da, MS/MS mass accuracy $\pm 0.6 \mathrm{Da}, 1$ accepted missed cleavage, carbamidomethylation of cysteine as a fixed modification, and oxidation of methionine as a possible one. After identification, each spot of the unaffected and affected fibroblast gels was compared to a calibrated reference gel of healthy human dermal fibroblasts of the Siena 2D-Database (www.bio-mol.unisi.it/cgi-bin/2d/2d_view_map.cgi?map= HUMAN_DERMAL_FIBROBLASTS_CL\&ac=all; data not shown).

Functional classification. The UniProt Knowledgebase (UniProtKB) was used as the central hub for the collection of functional information on identified proteins, with accurate, consistent, and rich annotation. Our dataset was also analyzed using the DAVID software to check for biological processes (expressed in terms GO) associated to proteins found in altered

Personal non-commercial use only. The Journal of Rheumatology Copyright $\subset$ C 2016. All rights reserved. 
abundance. DAVID software is a Web-accessible program that provides a complete set of bioinformatic tools for the functional characterization ${ }^{13}$. DAVID is able to help in understanding the biological significance hidden within a more or less extensive dataset ${ }^{14}$

Immunohistochemistry. The 3-mm punch biopsies were embedded in Tissue-Tek optimum cutting temperature compound (Sakura Finetek) fixation medium and frozen in isoprenalol cooled by liquid nitrogen, and then stored at $-70^{\circ} \mathrm{C}$ prior to analysis. Healthy skin samples (matched for age, sex, and site) that served as control references were taken from the Skin Biobank of the Dermatology Unit of University Hospital Le Scotte in Siena. Sections were cut at $5 \mu \mathrm{m}$ using a cryostat and then fixed in ice-cold acetone. Primary antibodies were used as follows: rabbit polyclonal anti-type I collagen (Col-1; Abcam), rabbit polyclonal anti-vimentin (anti-VIM; Abcam). Optimum dilution for the primary antibodies was determined by serial dilution. Bound primary antibodies were revealed using species-specific biotinylated secondary antibodies for $1 \mathrm{~h}$. Binding determined using the avidin/biotin complex system (Vector) and absolute eosinophil count substrate for peroxidase-red (Vector SK 4200). All subsequent steps were done at room temperature. Stained sections were imaged using Zeiss Axioscope.

RNA isolation and $q R T-P C R$. Healthy dermal fibroblasts that served as control references were taken from the Skin Biobank of the Dermatology Unit of University Hospital Le Scotte. Fibroblasts from lcSSc-affected subjects, unaffected subjects, and healthy controls were collected in TRIZOL reagent (Sigma-Aldrich). Total RNA was extracted following the manufacturer's instructions. The total RNA content of the samples was quantified by measuring the absorbance at $260 \mathrm{~nm}$, by Ultrospec 2000 spectrophotometer (Amersham Pharmacia Biotech). The RNA was then reverse-transcribed using random hexamer MultiScribe enzyme (Applied Biosystems Group). The StepOne Real-Time PCR System instrument (Applied Biosystems Group) with TaqMan chemistry was used to run qRT-PCR reactions. Two microliters of cDNA in a final volume of $20 \mu \mathrm{l}$ was amplified using the 20 $\times$ Assays-on-Demand gene expression assay mix (Applied Biosystems Group). Specific primers were designed on the basis of the reported sequences [Primer bank NCBI; Col-1: 5'-AGG GCC AAG ACG AAG ACA GT-3' (forward) and 5'-AGA TCA CGT CAT CGC ACA ACA-3' (reverse); VIM: 5'-GCAAAG ATT CCA CTT TGC GT-3' (forward) and 5'-GAAATT GCA GGA GGA GAT GC-3' (reverse)]. TaqMan probes, specific primers, and ribosomal 18S, selected as the housekeeping gene, were purchased from Applied Biosystems Group. The mRNA levels were normalized to those of $18 \mathrm{~S}$, and the relative mRNA levels after the treatment were calculated using the $\Delta \Delta \mathrm{CT}$ method $^{15}$.

Statistical analysis. For proteomic analysis, significant changes in abundance of proteins in the unaffected and affected fibroblast samples were determined based on a fold increase or decrease in protein level compared to standard values. Student's $t$ test was used for comparison of mean intensities between unaffected and affected fibroblasts, using a threshold of $p<0.05$ to establish the statistical significance of changes seen. Three technical replicates were performed. Real-time PCR statistics was performed using REST (Relative Expression Software Tool). Data are reported as mean fold change \pm SD of 3 technical replicates. Significance was set at $\mathrm{p}<0.05$.

\section{RESULTS}

Proteins of altered abundance in affected vs unaffected lcSSc fibroblasts. Composite images representative of the normal gel images are shown in Figure 1. The 37 protein spots for which unequivocal identities were obtained by MALDI-TOF MS are listed in Table 1. The comparison of lcSSc fibroblast gels with the healthy fibroblast reference gel found in the Siena 2D-Database revealed that lcSSc unaffected fibroblasts show a proteomic profile similar to healthy ones, but a different profile compared to lcSSc affected fibroblasts (data

\section{a}

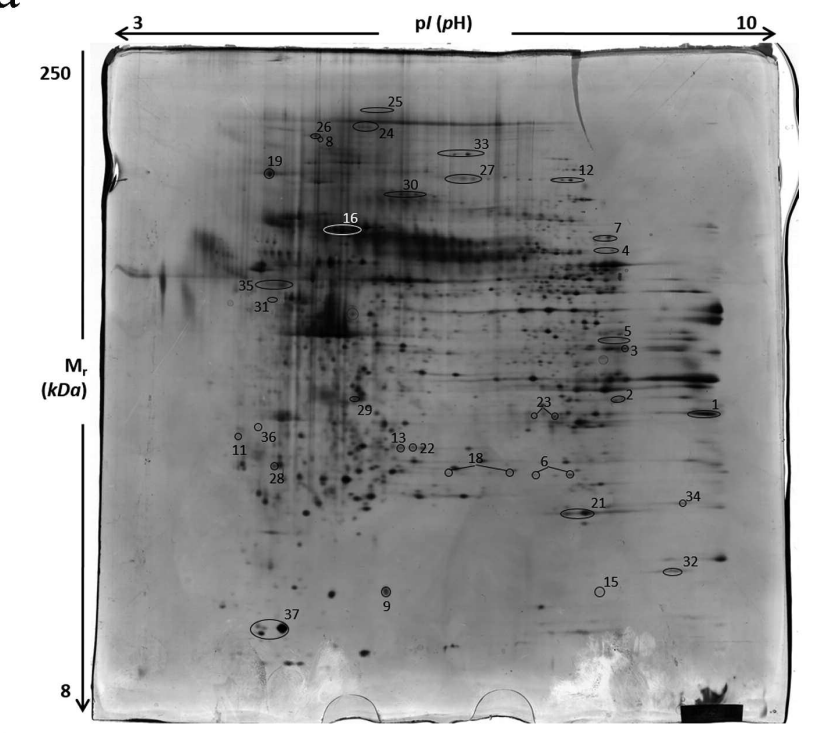

$\mathrm{b}$

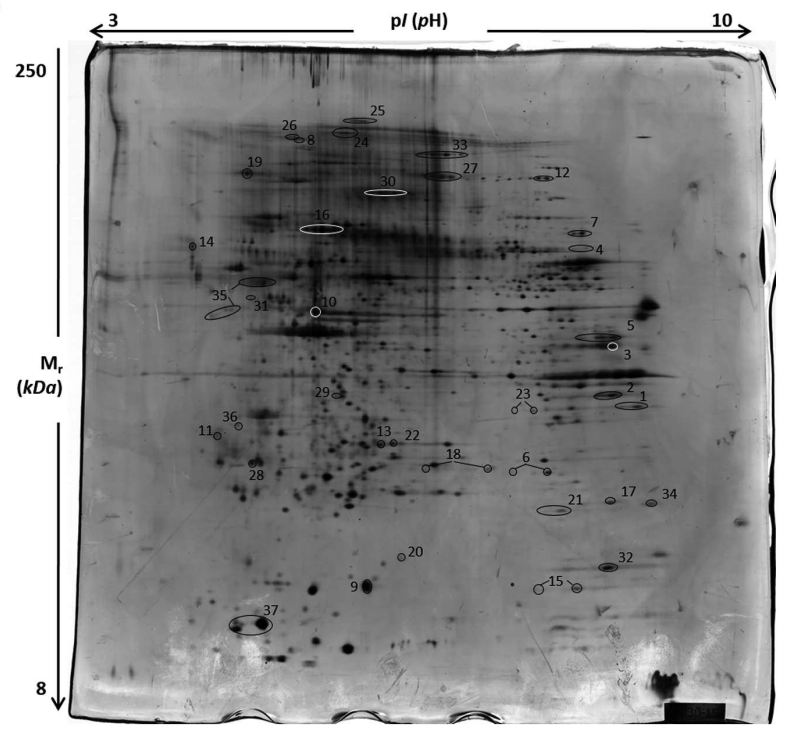

Figure 1. Proteomic analysis of 1cSSc unaffected (a) and affected (b) fibroblasts. Protein extracts of fibroblasts from unaffected skin samples ( $\mathrm{n}=5$ pooled) and affected ones ( $\mathrm{n}=5$ pooled) were separated by $2 \mathrm{DE}$ under $\mathrm{pH}$ gradients $\mathrm{pH} 3-10$. Mean intensity for each protein spot was determined for unaffected and affected samples. Proteins of altered abundance are outlined in gels and numbered from 1 to 37 . The corresponding proteins are listed in Table 1. Three technical replicates were performed. lcSSc: limited cutaneous systemic sclerosis; 2DE: 2-dimensional electrophoresis.

not shown). The UniProtKB functional classification of proteins found in altered abundance (Figure 2) indicates that lcSSc-affected fibroblasts show an impaired synthesis of proteins involved in cell organization $(27 \%)$, in stress response and cellular defense (22\%), in energy production $(19 \%)$, and in the organization of the extracellular matrix $(11 \%)$. Other functional classes less represented are the proteins involved in the synthesis and turnover $(8 \%)$, in signal transduction (3\%), associated to cellular homeostasis

Personal non-commercial use only. The Journal of Rheumatology Copyright @ 2016. All rights reserved 


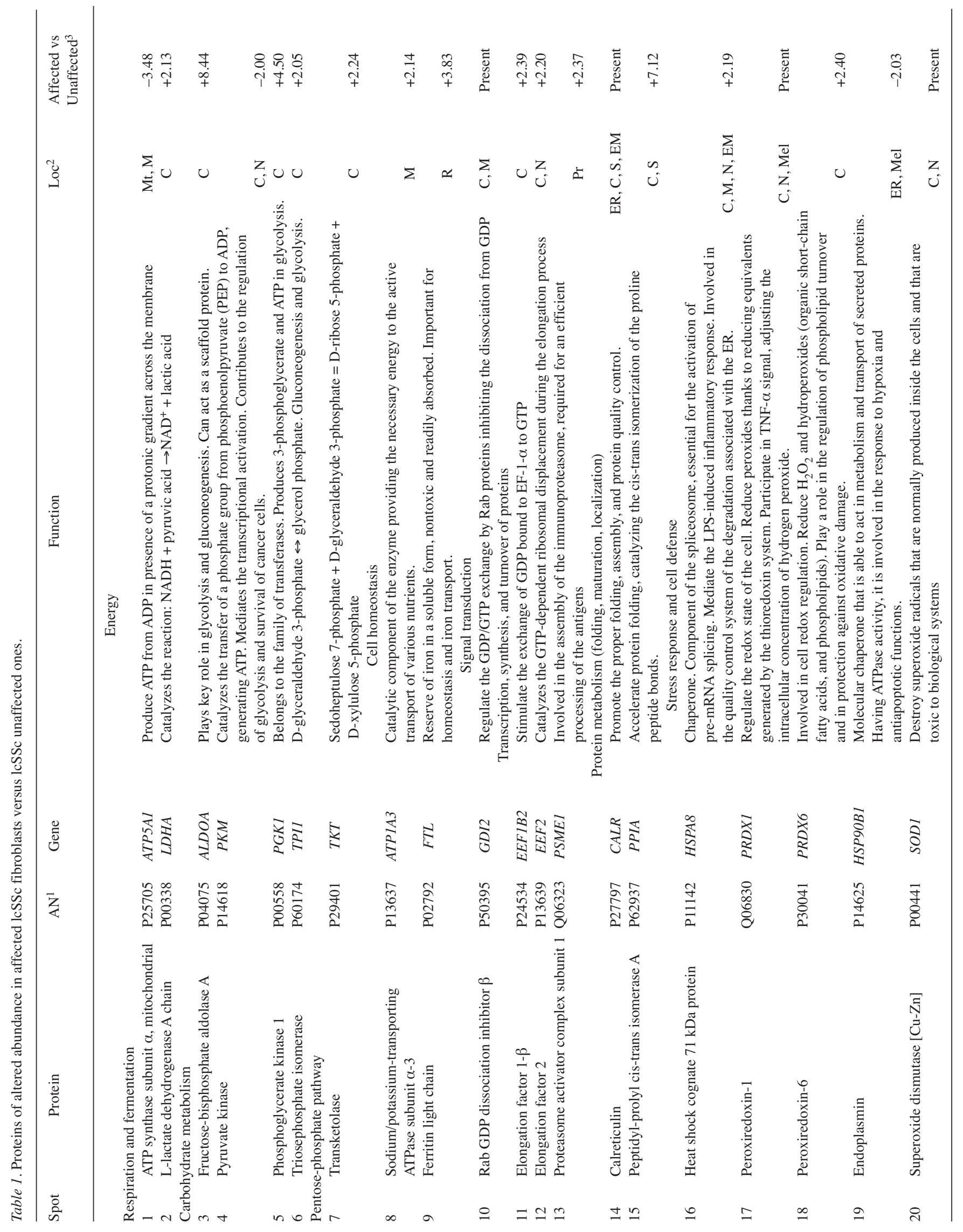

Personal non-commercial use only. The Journal of Rheumatology Copyright $\odot$ 2016. All rights reserved. 


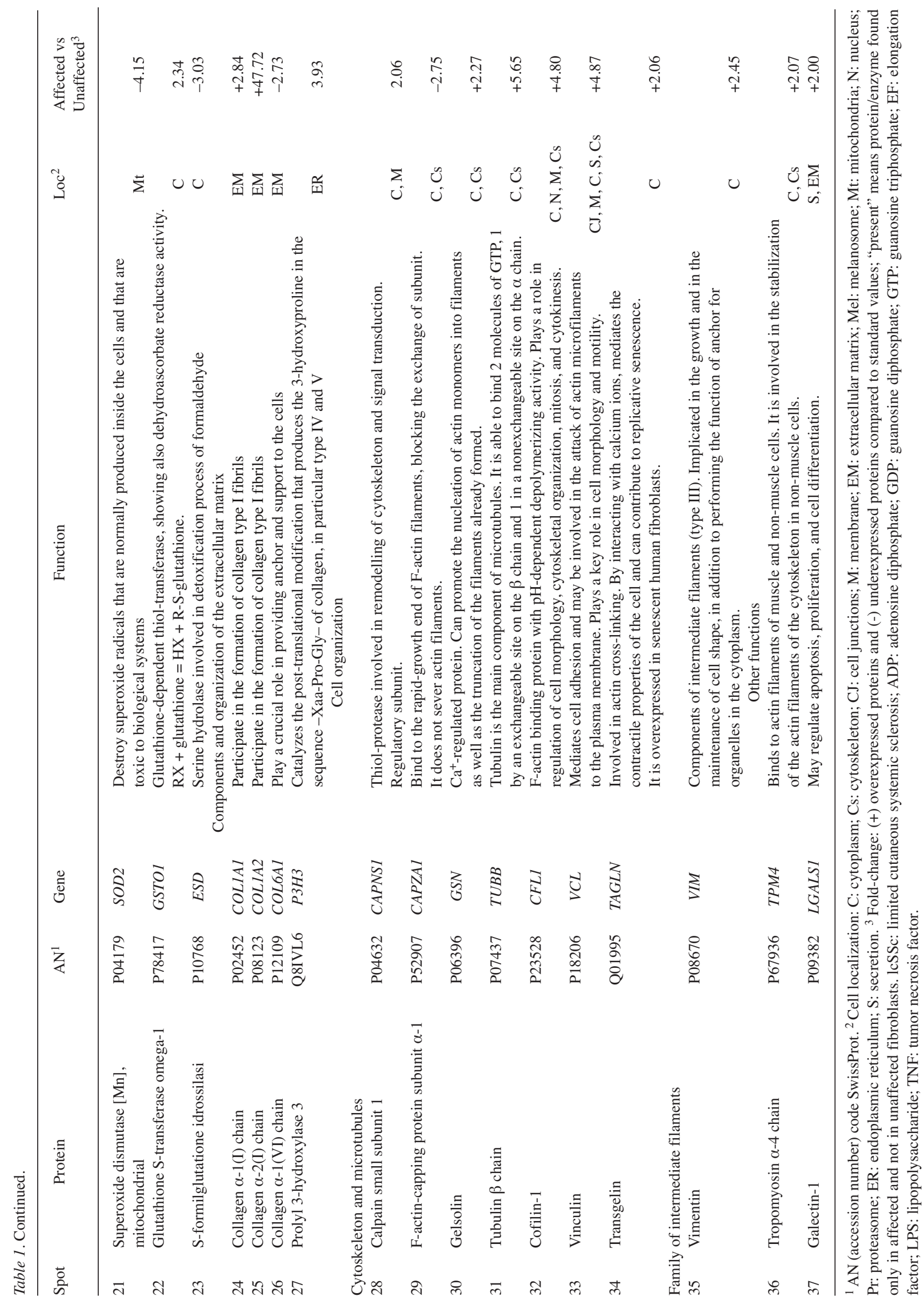

Personal non-commercial use only. The Journal of Rheumatology Copyright (c) 2016. All rights reserved. 


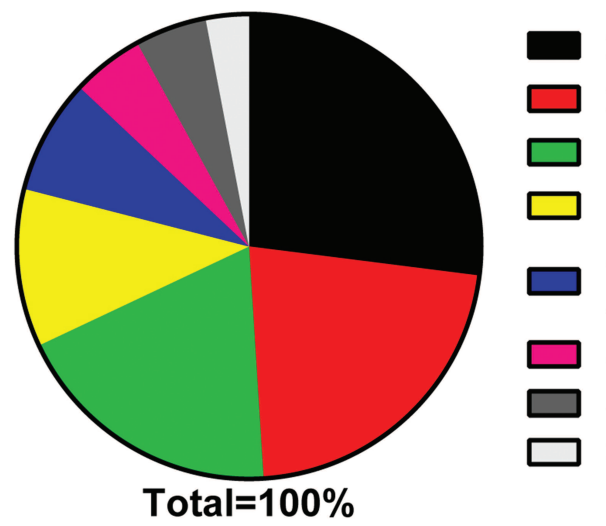

\section{$27 \%$ Organization of cytoskeleton \\ $22 \%$ Response to oxidative stress \\ $19 \%$ Energy metabolism \\ $11 \%$ Extracellular matrix remodelling \\ $8 \%$ Protein transcription, synthesis and turnover \\ $5 \%$ Cellular homeostasis \\ $5 \%$ Protein metabolism \\ $3 \%$ Signal transduction}

Figure 2. UniProt Knowledgebase functional classification of the biological processes associated to proteins found in altered abundance in lcSSc-affected fibroblasts. 1cSSc: limited cutaneous systemic sclerosis.

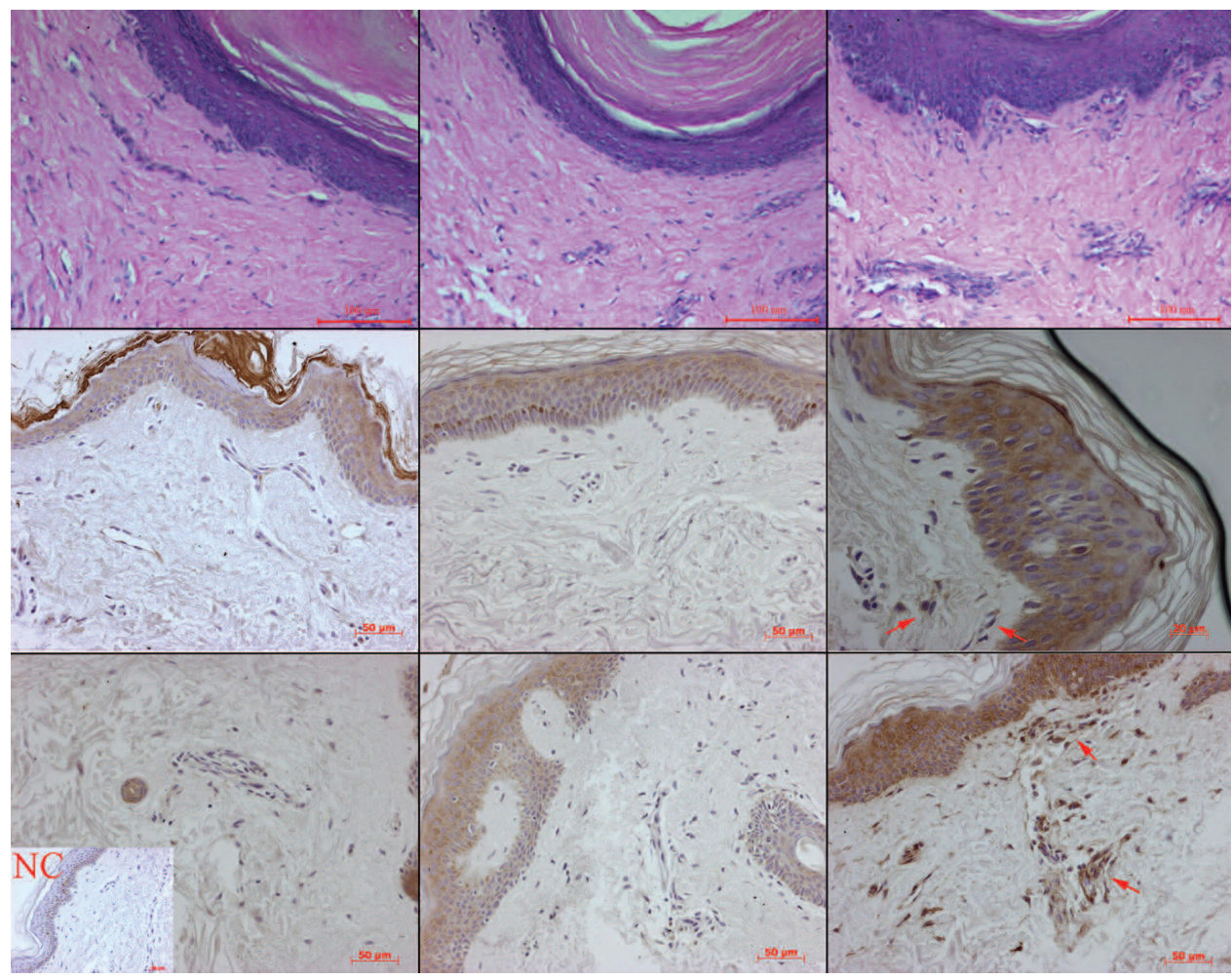

Figure 3. Histological H\&E staining (top panels) of healthy control (HC), lcSSc-unaffected, and lcSSc-affected skin samples, respectively. H\&E shows increased epidermal thickness of lcSSc-affected skin compared to lcSSc-unaffected and HC skin. Shown are type I collagen (Col-1; middle panels) and vimentin (VIM; bottom panels) stainings of healthy control, lcSSc-unaffected, and lcSSc-affected skin samples, respectively. In dermis of lcSSc-affected skin, fibroblasts (arrows) are strongly reactive to Col-1 and VIM, compared to lcSSc-unaffected and $\mathrm{HC}$ skin samples. NC (negative control) was obtained by replacing the primary antibodies with phosphate buffered saline. lcSSc: limited cutaneous systemic sclerosis.

$(5 \%)$, and in the metabolism of proteins $(5 \%)$. The functional classification analyzed by DAVID software is also reported in Table 2.

Immunohistochemistry of affected and unaffected lcSSc total skin samples and of healthy control skin. Figure 3 shows the H\&E (top panels), Col-1 (middle panels), and VIM (bottom panels) stainings of lcSSc affected, lcSSc unaffected, and healthy control skin samples. H\&E staining shows increased epidermal thickness of lcSSc affected skin compared to lcSSc unaffected and to healthy control skin. The dermis of affected lcSSc skin is strongly reactive to Col-1 and VIM, typical markers of activated myofibroblasts, compared to lcSSc unaffected and healthy control skin.

Results of $q R T-P C R$. Figure 4 shows qRT-PCR results related

Personal non-commercial use only. The Journal of Rheumatology Copyright (c) 2016. All rights reserved. 
Table 2. Cellular processes that are highly represented in proteins with impaired synthesis of affected lcSSc fibroblasts, selected by the DAVID software $(\mathrm{p}<0.05)$.

\begin{tabular}{|c|c|c|c|c|c|}
\hline Biological Process & Enrichment Score & $\mathrm{p}$ & Benjamini & Species No. & Proteins Involved in Biological Processes \\
\hline Glycolysis & 3.97 & $6.2 \mathrm{e}^{-6}$ & $4.7 \mathrm{e}^{-3}$ & 5 & $\begin{array}{l}\text { Triose-phosphate isomerase, fructose bisphosphate aldolase } \\
\text { A, lactate dehydrogenase, chain A, phosphoglycerate } \\
\text { kinase-1, pyruvate kinase }\end{array}$ \\
\hline Response to ROS & 2.87 & $1.3 \mathrm{e}^{-5}$ & $4.8 \mathrm{e}^{-3}$ & 5 & $\begin{array}{l}\text { Collagen } \alpha-1 \text { (type I), peroxiredoxin-1, peroxiredoxin- } 6 \text {, } \\
\text { superoxide dismutase } 1[\mathrm{Cu}-\mathrm{Zn}] \text {, superoxide dismutase } 2[\mathrm{Mn}]\end{array}$ \\
\hline Extracellular matrix & 1.75 & $1.8 \mathrm{e}^{-3}$ & $7.8 \mathrm{e}^{-2}$ & 6 & $\begin{array}{l}\text { Calreticulin, collagen } \alpha-1 \text { (type I), collagen } \alpha-2 \text { (type I), collagen } \\
\alpha-1 \text { (type IV), galectin- } 1 \text {, superoxide dismutase [Cu-Zn] }\end{array}$ \\
\hline Cell homeostasis & 1.65 & $1.6 \mathrm{e}^{-4}$ & $9.3 \mathrm{e}^{-3}$ & 8 & $\begin{array}{l}\text { Calreticulin, endoplasmin, ferritin, light chain, superoxide } \\
\text { dismutase } 1[\mathrm{Cu}-\mathrm{Zn}] \text {, superoxide dismutase } 2[\mathrm{Mn}] \text {, peroxiredoxin-1, } \\
\text { peroxiredoxin- } 6 \text {, fructose bisphosphate aldolase A }\end{array}$ \\
\hline
\end{tabular}

ROS: reactive oxygen species.
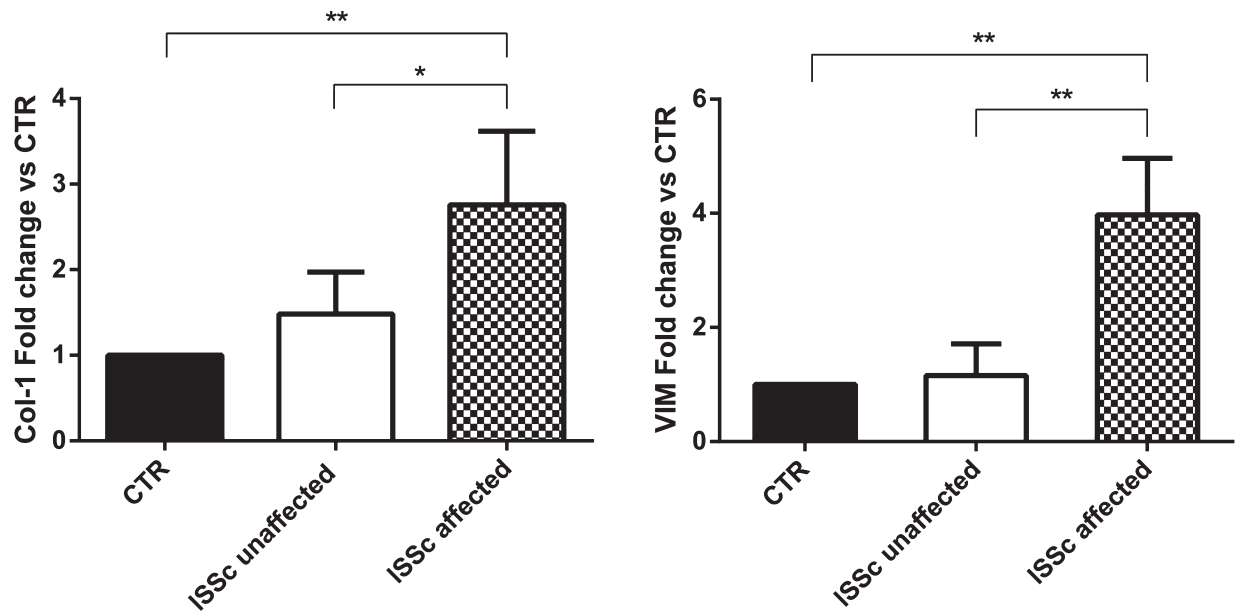

Figure 4. Type I collagen (Col-1, left panel) and vimentin (VIM, right panel) mRNA levels in control fibroblasts, lcSSc-unaffected, and lcSSc-affected fibroblasts. Data are reported as mean fold change \pm SD of 3 biological replicates. $* \mathrm{p}<0.05 . * * \mathrm{p}<0.01 .1 \mathrm{lSSc}(\mathrm{ISSc})$ : limited cutaneous systemic sclerosis; CTR: controls.

to Col-1 (left) and VIM (right) mRNA levels in lcSSc affected, lcSSc unaffected, and healthy control fibroblasts. Col-1 mRNA levels are statistically higher in lcSSc affected fibroblasts compared to lcSSc unaffected ones $(\mathrm{p}<0.05)$ and healthy controls $(\mathrm{p}<0.01)$. VIM mRNA levels are overexpressed in lcSSc affected fibroblasts compared to lcSSc unaffected ones $(\mathrm{p}<0.01)$ and healthy controls $(\mathrm{p}<0.01)$.

\section{DISCUSSION}

In our study, using proteomic analysis, we have identified changes in the pattern of protein expression in affected and unaffected skin fibroblasts of patients with $1 \mathrm{cSSc}$. Using 2DE followed by computer analysis, we demonstrated proteins present in altered abundance in the 2 skin biopsy samples. By using peptide profiling with MALDI-TOF MS, we were able to identify 30 proteins elevated and 7 proteins decreased in lcSSc-affected fibroblasts. Consistent with previous studies $^{16,17}$, proteins of altered abundance include proteins involved in extracellular matrix (ECM) production ${ }^{18}$, myofibroblast contractility ${ }^{19}$, response to oxidative stress $^{20}$, cellular homeostasis ${ }^{21}$, and energetic metabolism ${ }^{22}$. It is common knowledge that ECM protein overexpression plays a crucial role in the development and persistence of fibrosis in $\mathrm{SSc}^{23,24,25}$. Our proteomic data confirm the increase of ECM components in lcSSc-affected fibroblasts, in particular collagen $\alpha-1$ (type I) and collagen $\alpha-2$ (type I). It has been demonstrated that high type I collagen levels in SSc are correlated more with elevated mRNA steady-state levels rather than on the transcriptional activation of the relative genes: in fact, collagen mRNA transcripts in SSc fibroblasts were found to be more stable (half-life of $5 \mathrm{~h}$ ) compared with control cells (half-life of $2 \mathrm{~h})^{26}$. In this process, a crucial role is played by VIM filaments, whose association with collagen mRNA is responsible for the stabilization of the same 
collagen mRNA as a predominant mechanism for high collagen expression in fibrosis ${ }^{27}$. In fact, disruption of VIM filaments using the drug $\beta, \beta^{\prime}$-iminoditropionitrile reduces type I collagen expression, primarily owing to decreased stability of collagen $\mathrm{mRNA}^{27}$. The proteomics results related to upregulation of collagen and VIM in affected lcSSc fibroblasts were also confirmed by immunohistochemistry on total skin samples and by qRT-PCR analysis on fibroblasts. Therefore, upregulation of collagen and VIM play a fundamental role in the onset and evolution of cutaneous SSc changes: moreover, we believe that these findings will serve as a rationale for targeting VIM and collagen in the development of novel antifibrotic therapies in SSc. The upregulation of VIM is followed in lcSSc-affected fibroblasts by overexpression of calpain, gelsolin, $\beta$-tubulin, cofilin- 1 , vinculin, and tropomyosin, all proteins involved in cytoskeletal organization $28,29,30,31,32,33$. This intracellular network is responsible for the physical ECM attachment to the nucleus through integrin receptors, originating a mechanosensory and signaling apparatus ${ }^{34}$. In lcSSc-affected fibroblasts we found no alteration of the number and thickness of actin filaments, suggesting that the increased cell flexibility might be due to the different structure of microtubules or to the cytoskeletal reorganization ${ }^{35}$.

Further, by the analysis of mechanical properties, it has been demonstrated that SSc-affected fibroblasts are less rigid compared to healthy ones, and so they become more sensitive to mechanical stimuli inducing production of ECM components and less sensitive to mechanical inhibitory stimuli ${ }^{36}$. In fact, in vivo, fibroblast removal during the wound healing and scarring processes is induced by apoptosis through inhibitory mechanical stimulation, such as the contraction of the collagen matrix mediated by intracellular integrin-dependent signaling ${ }^{36}$. In this perspective, because SSc-affected fibroblasts are less rigid and so less sensitive to mechanical inhibitory control, they become resistant to apoptosis ${ }^{35}$. This study demonstrated altered abundance of proteins involved not only in ECM production and myofibroblast contractility, but also in oxidative stress response, as recently reported $37,38,39$. SSc- affected fibroblasts produce high amounts of reactive oxygen species (ROS), as a consequence of the hyperactivation of the nicotinamide adenine dinucleotide phosphate hydrogen (NADPH) oxidase enzymatic system ${ }^{40}$. These high ROS levels induce fibroblast differentiation into myofibroblast phenotype and consequently type I collagen synthesis ${ }^{41}$. We found overexpression of peroxiredoxin-1 (PRDX1), PRDX6, and superoxide dismutase 1 (SODM 1) in lcSSc-affected fibroblasts: these proteins represent the mechanisms of antioxidant defense by fibroblasts to counteract the increase of intracellular ROS, as evidenced in literature ${ }^{41}$. On the contrary, the underexpressed levels of SODM 2, found in lcSSc-affected fibroblasts, confirm the hyperactivation of the NADPH oxidase system ${ }^{42}$.

Our findings demonstrated that dermal fibroblasts in affected lcSSc skin are in an activated myofibroblast state, and contribute in an amplifying profibrotic loop leading to $\mathrm{SSc}$ tissue remodeling. The proteomic investigation revealed that fibroblasts from affected and unaffected skin of the same patient with lcSSc act as 2 distinct entities within the same body, and that lcSSc-unaffected fibroblasts and total skin samples share similar behavior with fibroblasts from healthy controls and total skin samples. Therefore, future treatment strategies for established skin fibrosis in the limited form of the disease should take into consideration the 2 distinct fibroblast entities and should be addressed only against the activated myofibroblast phenotype of the affected skin, perhaps acting on the VIM-collagen system.

\section{REFERENCES}

1. Denton CP. Advances in pathogenesis and treatment of systemic sclerosis. Clin Med 2016;16:55-60.

2. Van Den Hoogen F, Khanna D, Fransen J, Johnson SR, Baron M, Tyndall A, et al. 2013 classification criteria for systemic sclerosis an American College of Rheumatology/European League against Rheumatism collaborative initiative. Arthritis Rheum 2013; 65:2737-47.

3. Jimenez SA, Hitraya E, Varga J. Pathogenesis of scleroderma. Collagen. Rheum Dis Clin North Am 1996;22:647-74.

4. Del Galdo F, Shaw MA, Jimenez SA. Proteomic analysis identification of a pattern of shared alterations in the secretome of dermal fibroblasts from systemic sclerosis and nephrogenic systemic fibrosis. Am J Pathol 2010;177:1638-46.

5. Clements PJ, Lachenbruch PA, Seibold JR, Zee B, Steen VD, Brennan P, et al. Skin thickness score in systemic sclerosis: an assessment of interobserver variability in 3 independent studies. J Rheumatol 1993;20:1892-6.

6. Desbois AC, Cacoub P. Systemic sclerosis: An update in 2016. Autoimmun Rev 2016;15:417-26.

7. Bradford MM. A rapid and sensitive method for the quantitation of microgram quantities of protein utilizing the principle of protein-dye binding. Anal Biochem 1976;72:248-54.

8. Bjellqvist B, Pasquali C, Ravier F, Sanchez JC, Hochstrasser D. A nonlinear wide-range immobilized $\mathrm{pH}$ gradient for two-dimensional electrophoresis and its definition in a relevant $\mathrm{pH}$ scale. Electrophoresis 1993;14:1357-65.

9. Hochstrasser DF, Harrington MG, Hochstrasser AC, Miller MJ, Merril CR. Methods for increasing the resolution of two-dimensional protein electrophoresis. Anal Biochem 1988;173:424-35.

10. Oakley BR, Kirsch DR, Morris NR. A simplified ultrasensitive silver stain for detecting proteins in polyacrylamide gels. Anal Biochem 1980;105:361-3.

11. Hochstrasser DF, Patchornik A, Merril CR. Development of polyacrylamide gels that improve the separation of proteins and their detection by silver staining. Anal Biochem 1988;173:412-23.

12. Hellman U, Wernstedt C, Gonez J, Heldin CH. Improvement of an "In-Gel" digestion procedure for the micropreparation of internal protein fragments for amino acid sequencing. Anal Biochem 1995;224:451-5.

13. Huang DW, Sherman BT, Lempicki RA. Systematic and integrative analysis of large gene lists using DAVID Bioinformatics Resources. Nature Protoc 2009;4:44-57.

14. Huang DW, Sherman BT, Lempicki RA. Bioinformatics enrichment tools: paths toward the comprehensive functional analysis of large gene lists. Nucleic Acids Res 2009;37:1-13.

15. Livak KJ, Schmittgen TD. Analysis of relative gene expression data

Personal non-commercial use only. The Journal of Rheumatology Copyright (C) 2016. All rights reserved. 
using real-time quantitative PCR and the 2(-Delta Delta C(T)) Method. Methods 2001;25:402-8.

16. Aden N, Shiwen X, Aden D, Black C, Nuttall A, Denton CP, et al. Proteomic analysis of scleroderma lesional skin reveals activated wound healing phenotype of epidermal cell layer. Rheumatology 2008;47:1754-60.

17. Davidson JM. Proteomic revelations. J Invest Dermatol 2014;134:2301-2.

18. Cutolo M, Sulli A, Pizzorni C, Paolino S, Smith V. Systemic sclerosis: markers and targeted treatments. Acta Reumatol Port 2016;41:18-25

19. Abraham DJ, Eckes B, Rajkumar V, Krieg T. New developments in fibroblast and myofibroblast biology: implications for fibrosis and scleroderma. Curr Rheumatol Rep 2007;9:136-43.

20. Svegliati S, Cancello R, Sambo P, Luchetti M, Paroncini P, Orlandini $\mathrm{G}$, et al. Platelet-derived growth factor and reactive oxygen species (ROS) regulate Ras protein levels in primary human fibroblasts via ERK1/2. Amplification of ROS and Ras in systemic sclerosis fibroblasts. J Biol Chem 2005;280:36474-82.

21. Tomcik M, Palumbo-Zerr K, Zerr P, Avouac J, Dees C, Sumova B, et al. S100A4 amplifies TGF- $\beta$-induced fibroblast activation in systemic sclerosis. Ann Rheum Dis 2015;74:1748-55

22. Cong L, Xia ZK, Yang RY. Targeting the TGF- $\beta$ receptor with kinase inhibitors for scleroderma therapy. Arch Pharm 2014;347:609-15.

23. Stern EP, Denton CP. The pathogenesis of systemic sclerosis. Rheum Dis Clin North Am 2015;41:367-82.

24. Leask A. Matrix remodeling in systemic sclerosis. Semin Immunopathol 2015;37:559-63.

25. Gilbane AJ, Denton CP, Holmes AM. Scleroderma pathogenesis: a pivotal role for fibroblasts as effector cells. Arthritis Res Ther 2013;15:215.

26. Eckes B, Mauch C, Hüppe G, Krieg T. Differential regulation of transcription and transcript stability of pro-alpha 1(I) collagen and fibronectin in activated fibroblasts derived from patients with systemic scleroderma. Biochem J 1996;315:549-54.

27. Challa AA, Stefanovic B. A novel role of vimentin filaments: binding and stabilization of collagen mRNAs. Mol Cell Biol 2011;31:3773-89.

28. Nassar D, Letavernier E, Baud L, Aractingi S, Khosrotehrani K. Calpain activity is essential in skin wound healing and contributes to scar formation. PLoS One 2012;7:e37084.

29. Chan MW, Arora PD, Bozavikov P, McCulloch CA. FAK, PIP5KIgamma and gelsolin cooperatively mediate force-induced expression of alpha-smooth muscle actin. J Cell Sci 2009; $122: 2769-81$
30. Feghali CA, Wright TM. Identification of multiple, differentially expressed messenger RNAs in dermal fibroblasts from patients with systemic sclerosis. Arthritis Rheum 1999;42:1451-7.

31. Courtemanche N, Gifford SM, Simpson MA, Pollard TD, Koleske AJ. Abl2/Abl-related gene stabilizes actin filaments, stimulates actin branching by actin-related protein $2 / 3$ complex, and promotes actin filament severing by cofilin. J Biol Chem 2015;290:4038-46.

32. Lai YZ, Chen J, Lin S. Effects of morphology of the microgroove titanium surface on expression of vinculin in human gingival fibroblasts. Shanghai Kou Qiang Yi Xue 2015;24:182-7.

33. Prunotto M, Bruschi M, Gunning P, Gabbiani G, Weibel F, Ghiggeri $\mathrm{GM}$, et al. Stable incorporation of $\alpha$-smooth muscle actin into stress fibers is dependent on specific tropomyosin isoforms. Cytoskeleton 2015;72:257-67.

34. Ingber DE. Cellular mechanotransduction: putting all the pieces together again. FASEB J 2006;20:811-27.

35. Reich A, Meurer M, Eckes B, Friedrichs J, Muller DJ. Surface morphology and mechanical properties of fibroblasts from scleroderma patients. J Cell Mol Med 2009;13:1644-52.

36. Nho RS, Xia H, Kahm J, Kleidon J, Diebold D, Henke CA. Role of integrin-linked kinase in regulating phosphorylation of Akt and fibroblast survival in type I collagen matrices through a beta1 integrin viability signaling pathway. J Biol Chem 2005; 280:26630-9.

37. Bourji K, Meyer A, Chatelus E, Pincemail J, Pigatto E, Defraigne $\mathrm{JO}$, et al. High reactive oxygen species in fibrotic and nonfibrotic skin of patients with diffuse cutaneous systemic sclerosis. Free Radic Biol Med 2015;87:282-9.

38. Piera-Velazquez S, Makul A, Jiménez SA. Increased expression of NAPDH oxidase 4 in systemic sclerosis dermal fibroblasts: regulation by transforming growth factor $\beta$. Arthritis Rheumatol 2015;67:2749-58.

39. Amico D, Spadoni T, Rovinelli M, Serafini M, D'Amico G, Campelli $\mathrm{N}$, et al. Intracellular free radical production by peripheral blood T lymphocytes from patients with systemic sclerosis: role of NADPH oxidase and ERK1/2. Arthritis Res Ther 2015;17:68.

40. Sambo P, Amico D, Giacomelli R, Matucci-Cerinic M, Salsano F, Valentini G, et al. Intravenous $\mathrm{N}$-acetylcysteine for treatment of Raynaud's phenomenon secondary to systemic sclerosis: a pilot study. J Rheumatol 2001;28:2257-62.

41. Grygiel-Górniak B, Puszczewicz M. Oxidative damage and antioxidative therapy in systemic sclerosis. Mediators Inflamm 2014;2014:389582.

42. Dikalov S. Cross talk between mitochondria and NADPH oxidases. Free Radic Biol Med 2011;51:1289-301. 